\title{
Urothelial Carcinoma of the Upper Urinary Tract That Becomes Resectable After Neoadjuvant Chemotherapy: A Case Report and Review of the Literature
}

\author{
(D) Mustafa Dinçkal, (1) Fuat Kızılay, (1) Serdar Kalemci, (1) Adnan Şimşir \\ Ege University Faculty of Medicine, Department of Urology, İzmir, Turkiye
}

\begin{abstract}
Upper tract urothelial carcinoma (UTUC) is less common than bladder cancer, but its incidence is increasing. Neoadjuvant chemotherapy (NAC) has been the treatment focus for locally invasive and high-grade UTUC. Herein, we aimed to present a case of a locally advanced non-metastatic UTUC, which was thought to be unresectable due to local invasion, but surgically treated successfully after NAC. A 64-year-old male patient was admitted to another hospital because of right flank pain, which was not accompanied by macroscopic haematuria. He did not have comorbidities in his anamnesis, but he had a history of smoking 40 packs/year. A locally invasive right kidney tumour was detected in cross-sectional imaging performed at another hospital. He underwent surgery in that hospital, but radical nephroureterectomy could not be performed because of local invasion. He presented to our urology department. He was subsequently started with cisplatin-based NAC, which led to the resolution of local invasion. After NAC, right radical nephroureterectomy and ipsilateral bladder cuff excision with subcostal and Gibson incisions were performed. No signs of mass invasion or lymph node involvement were detected intraoperatively. He was examined 3 months after surgery. On cystourethroscopy, the bladder was normal and cytology was benign. No recurrence or metastasis was detected on the whole-body computed tomography. NAC is one of the valuable multimodal treatment options, enables surgery in locally invasive UTUC and contributes positively to survival rate.
\end{abstract}

Keywords: Oncology, urooncology, urothelial carcinoma, nephrectomy, nephroureterectomy, neoadjuvant chemotherapy

\section{Introduction}

Upper tract urothelial carcinoma (UTUC) accounts for 5\% of urothelial cancers. Although UTUC is less common than bladder cancer, its incidence is increasing recently $(1,2)$. Radical nephroureterectomy (RNU) and excision of the ipsilateral bladder cuff is the standard surgical procedure for the treatment of UTUC, and surgical treatment can often be curative alone (3). In recent years, organ-sparing approaches have also been considered in some selected cases. Although the standard treatment is RNU, recurrence rates after the surgical approach in locally advanced diseases are quite high, and growing evidence shows the role of both adjuvant chemotherapy (AC) and neoadjuvant chemotherapy (NAC) $(4,5)$. Especially in locally advanced disease, the pathological downstaging (PD) rates by NAC have been shown to contribute positively to survival (6).

Herein, we will present a case of a locally advanced nonmetastatic UTUC, which was thought to be unresectable due to local invasion but successfully surgically treated after NAC.

\section{Case Presentation}

A 64-year-old male patient was admitted to another hospital because of right flank pain, which was not accompanied by macroscopic haematuria. He did not have comorbidities in his anamnesis, but he had a history of smoking 40 packs/year.

Computed tomography urography was performed after ultrasonography and detected a mass in the right kidney. A $60 \times 85 \times 100 \mathrm{~mm}^{3}$ infiltrative mass extending to the renal pelvis was detected in the upper and middle calices of the kidney (Figure 1). At the level of the renal hilus, two lymph nodes of $25 \mathrm{~mm}$ and $26 \mathrm{~mm}$ in diameter were observed anterior to the inferior vena cava. The mass infiltrated the perirenal fat. No metastatic focus or extra pathology was detected in chest computed tomography.

On cystourethroscopy, no tumour was observed in the bladder, so RNU was arranged in the first hospital, but RNU could not be performed. His operation report stated that the abdominal wall muscles were cut through the right subcostal incision and

Correspondence: Mustafa Dinçkal MD, Ege University Faculty of Medicine, Department of Urology, İzmir, Turkiye Phone: +90 2323902500 E-mail: drmustafadinckal@gmail.com ORCID-ID: orcid.org/0000-0003-4905-1602 Received: 27.07 .2020 Accepted: 12.11 .2020

Cite this article as: Dinçkal M, Kızılay F, Kalemci S, Șimșir A. Urothelial Carcinoma of the Upper Urinary Tract That Becomes Resectable After Neoadjuvant Chemotherapy: A Case Report and Review of the Literature. J Urol 2021;8(1):56-68.

${ }^{\circ}$ Copyright 2020 by the Association of Urological Surgery / Journal of Urological Surgery published by Galenos Publishing House. 
entered into the abdominal cavity. The right colon was released, and the right kidney was reached. The surgery was terminated because renal pedicle release was not possible due to inferior invasion of the vena cava.

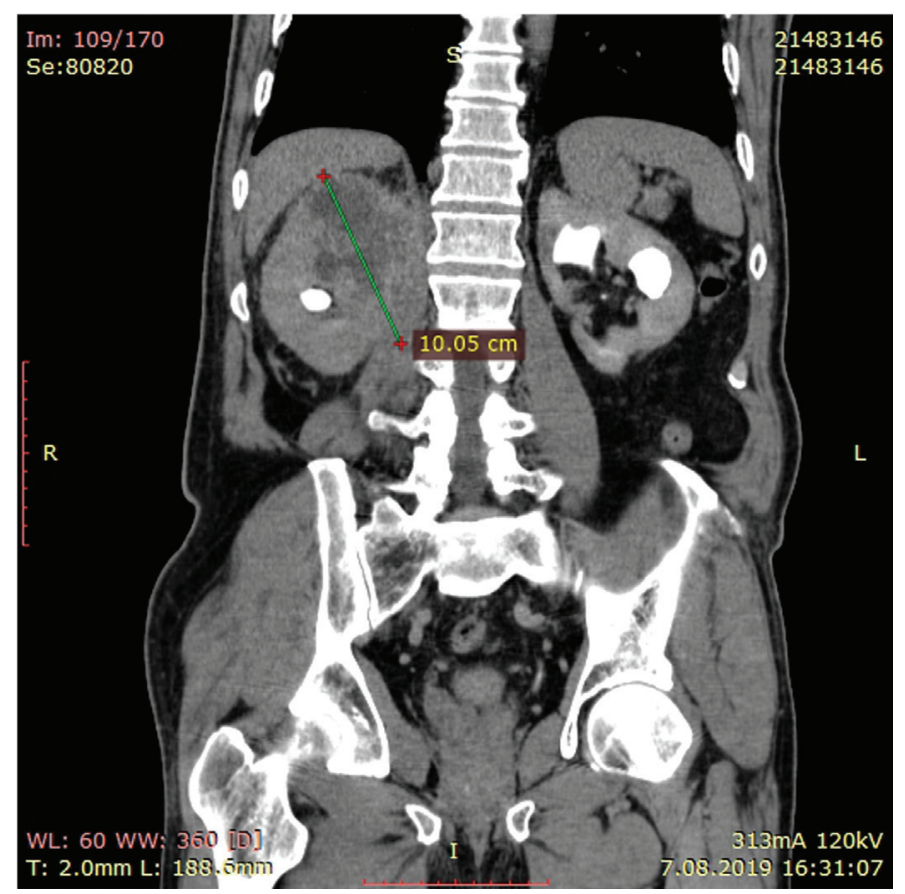

Figure 1. Before neoadjuvant chemotherapy

He was referred to our urology department, and a renal biopsy was then performed. NAC was initiated because his renal biopsy histopathological examination finding was compatible with urothelial carcinoma and a local invasive mass was found in the right kidney. The patient received two cycles of gemcitabine $\left(1,000 \mathrm{mg} / \mathrm{m}^{2}\right)$ and cisplatin $\left(80 \mathrm{mg} / \mathrm{m}^{2}\right)$. Magnetic resonance imaging was performed for restaging after NAC, which revealed that the mass in the right kidney regressed to $53 \times 60 \times 70 \mathrm{~mm}^{3}$, and the two pre-existing lymph nodes disappeared (Figure 2).

Right RNU and ipsilateral bladder cuff excision with subcostal and Gibson incisions was performed. No signs of mass invasion or lymph node involvement were detected intraoperatively (Figure 3). Histopathological diagnosis was interpreted as pT4 urothelial carcinoma, and the surgical margins were clear (Figure 4).

The patient was examined 3 months after surgery. On cystourethroscopy, the bladder was normal and cytology was benign. No recurrence or metastasis was detected on the wholebody computed tomography. Written informed consent was obtained from the patient.

\section{Discussion}

Evidence on the effectiveness of NAC is based on the results of AC treatment for bladder cancer (1). Compared with AC, NAC may be

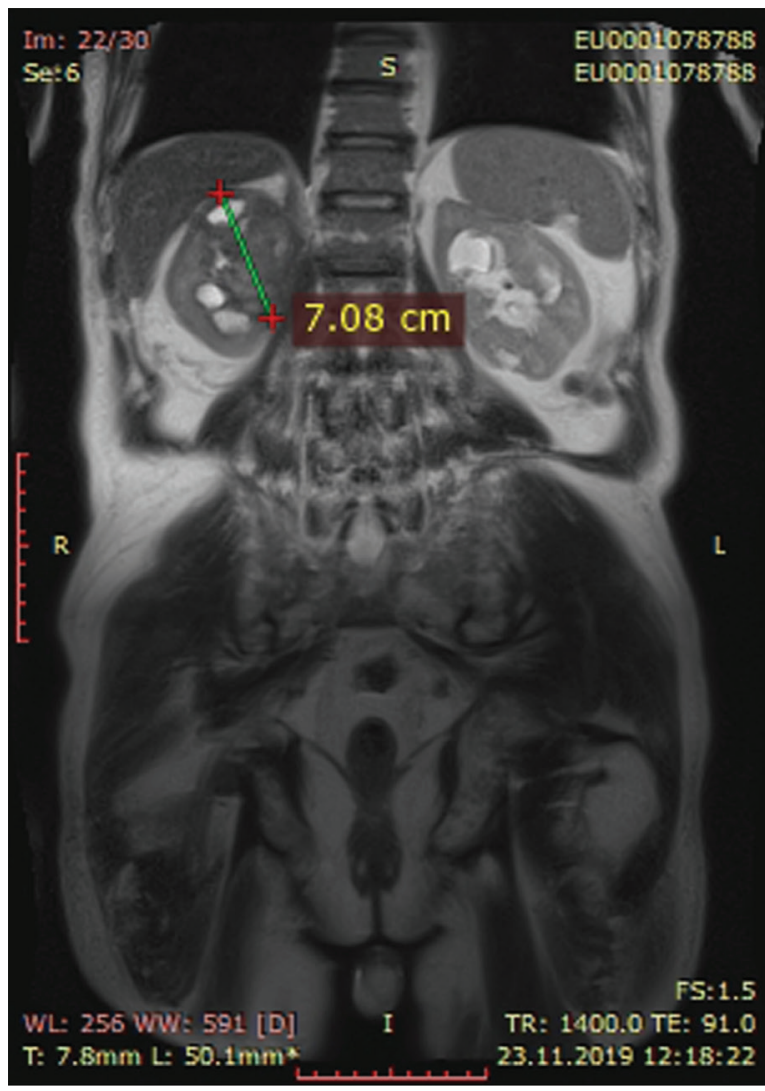

Figure 2. After neoadjuvant chemotherapy

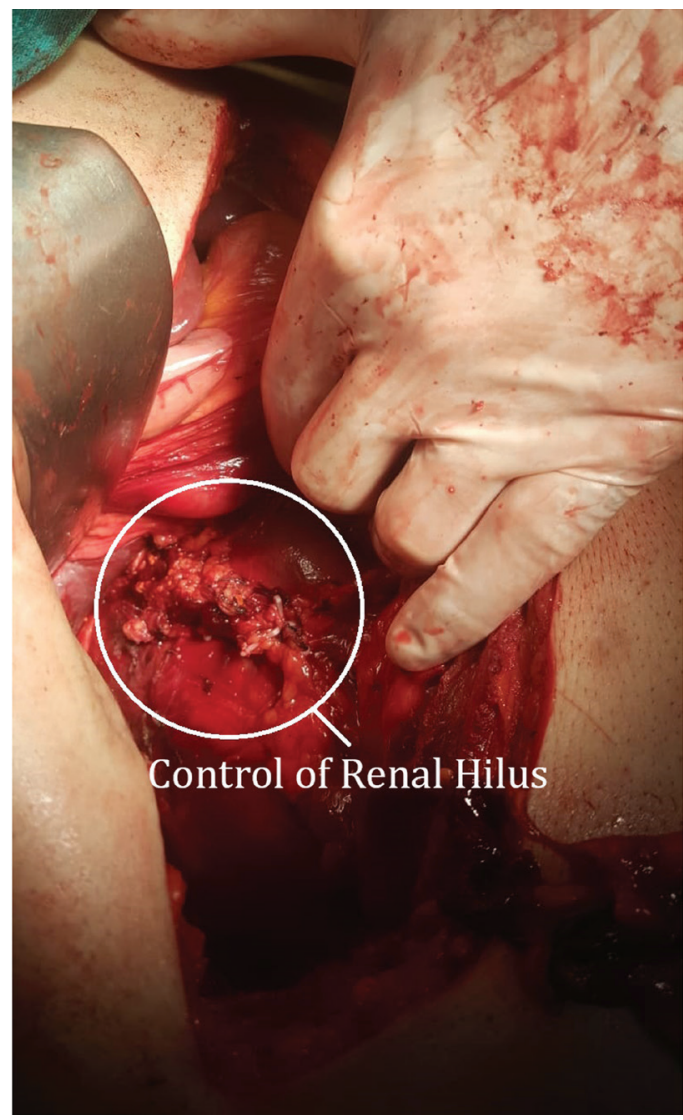

Figure 3. Renal hilus control 


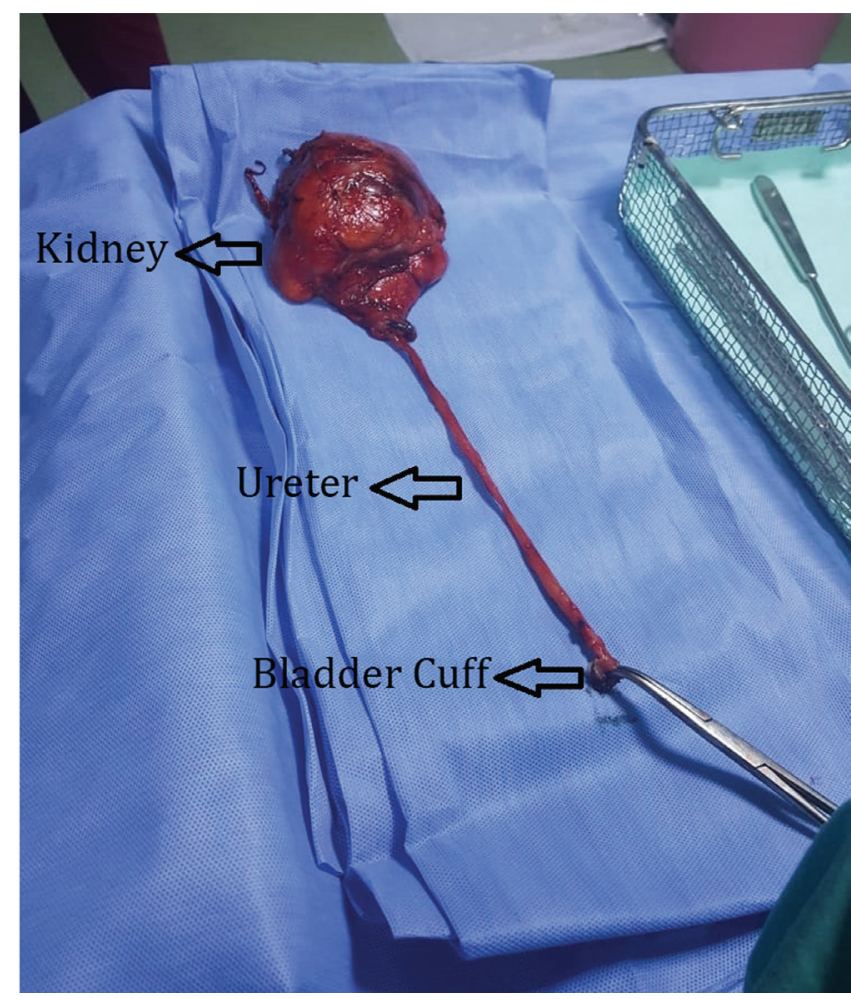

Figure 4. Postoperative view of specimen

considered as a more effective treatment option for UTUC, because some patients cannot receive cisplatin-based AC due to postoperative kidney function impairment (7-9). Cisplatinbased NAC can result in higher pathological complete response (PCR) and PD rates than other chemotherapy regimens, but creatinine clearance must be $>50 \mathrm{~mL} / \mathrm{min}$ for the suitability of this treatment (10). Therefore, preoperative chemotherapy treatment may be more advantageous.

Although UTUC is morphologically similar to bladder cancer, different phenotypically and genotypically malignancies exist because of the high rate of locally advanced disease development (11). The 5-year overall survival (OS) rates of locally invasive UTUC stage T2 and T3 were $73 \%$ and $40 \%$, respectively. The median survival time was 6 months in T4 stage (12). Although RNU is the standard treatment for high-grade and locally advanced UTUC, its recurrence rate is quite high. Thus, NAC as treatment for bladder cancer might be considered to treat occult micrometastasis and provide PDs (8). In a recent

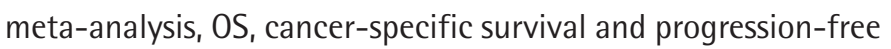
survival in patients receiving NAC were 57\%, 59\% and 45\%, respectively, and PDs increased by 4.76 -fold in the NAC-treated group (13). Compared with PCR after NAC, the PD is a stronger prognostic factor for survival rates $(6,14)$. In a prospective study investigating the pathological response to NAC, the PCR rate was $14 \%$ and the PD was $60 \%$ (10). In our case, the pathological lymph nodes disappeared in magnetic resonance imaging performed after NAC.
As stated in the European Association of Urology guideline, the NAC regimen used in bladder cancer is also effective in the treatment of UTUC (15). However, some patients may lose their chance to undergo surgery, or surgical treatment may be delayed due to the toxicity of chemotherapy after NAC (16). In a study of 61 patients who received NAC for high-grade UTUC, only one patient developed sepsis, but he eventually recovered, and none of the patients had lost their chances for surgery and they have progressive disease (17).

NAC cycle is still controversial for UTUC. A delay of $>90$ days for radical cystectomy was reported to be associated with a poor prognosis (18). In studies investigating NAC for UTUC, the tumour response was evaluated after two cycles of NAC within 90 days, in line with the recommendations for invasive bladder cancer treatment, and surgery was planned $(19,20)$. Patients with insufficient tumour response (stable or progressive disease) received three or four cycles of NAC (20). Currently, only a few prospective randomised trials are underway for UTUC $(21,22)$. These studies will shed light on NAC for UTUC. In our case, reimaging was performed to evaluate tumour response after two cycles of NAC. After obtaining a tumour response, we decided to perform surgery.

Despite these data, NAC, which has level 1 evidence in locally advanced BC, has not been the standard treatment in locally advanced and high-grade UTUC, owing to the lack of prospective randomised studies (23).

Upon presentation to our department, we considered that the patient was an RNU candidate, but the case was judged as unresectable due to local invasion. As a result, we decided to initiate NAC. Thereafter, local invasion was resolved, and surgery was feasible and successfully performed. NAC is one of the valuable multimodal treatment options that should be kept in mind because it facilitates surgery in locally invasive UTUC and contributes positively to survival rate.

\section{Ethics}

Informed Consent: Written informed consent was obtained from the patient.

Peer-review: Externally peer-reviewed.

\section{Authorship Contributions}

Surgical and Medical Practices: M.D., F.K., S.K., A.Ş., Concept: M.D., Design: M.D., Data Collection or Processing: M.D., Analysis or Interpretation: F.K., S.K., A.Ş., Literature Search: M.D., Writing: M.D.

Conflict of Interest: No conflict of interest was declared by the authors. 
Financial Disclosure: The authors declared that this study received no financial support.

\section{References}

1. Raman JD, Scherr DS. Management of patients with upper urinary tract transitional cell carcinoma. Nat Clin Pract Urol 2007;4:432-443.

2. Zigeuner $R$, Pummer K. Urothelial carcinoma of the upper urinary tract: surgical approach and prognostic factors. Eur Urol 2008;53:720-731.

3. Margulis V, Shariat SF, Matin SF, Kamat AM, Zigeuner R, Kikuchi E, Lotan Y, Weizer A, Raman JD, Wood CG; Upper Tract Urothelial Carcinoma CollaborationThe Upper Tract Urothelial Carcinoma Collaboration. Outcomes of radical nephroureterectomy: a series from the Upper Tract Urothelial Carcinoma Collaboration. Cancer 2009;115:1224-1233.

4. Rouprêt $M$, Babjuk $M$, Compérat $E$, Zigeuner $R$, Sylvester RJ, Burger $M$, Cowan NC, Gontero P, Van Rhijn BWG, Mostafid AH, Palou J, Shariat SF. European Association of Urology Guidelines on Upper Urinary Tract Urothelial Carcinoma: 2017 Update. Eur Urol 2018;73:111-122.

5. Kang $\mathrm{M}$, Jeong $\mathrm{CW}, \mathrm{Kwak} \mathrm{C}, \mathrm{Kim} \mathrm{HH}, \mathrm{Ku} \mathrm{JH}$. The characteristics of recurrent upper tract urothelial carcinoma after radical nephroureterectomy without bladder cuff excision. Yonsei Med J 2015;56:375-381.

6. Martini A, Daza J, Poltiyelova E, Gul Z, Heard JR, Ferket BS, Waingankar N, Galsky MD, Sfakianos JP. Pathological downstaging as a novel endpoint for the development of neoadjuvant chemotherapy for upper tract urothelial carcinoma. BJU Int 2019;124:665-671.

7. Leow JJ, Martin-Doyle W, Fay AP, Choueiri TK, Chang SL, Bellmunt J. A systematic review and meta-analysis of adjuvant and neoadjuvant chemotherapy for upper tract urothelial carcinoma. Eur Urol 2014;66:529541.

8. Tabayoyong W, Li R, Gao J, Kamat A. Optimal Timing of Chemotherapy and Surgery in Patients with Muscle-Invasive Bladder Cancer and Upper Urinary Tract Urothelial Carcinoma. Urol Clin North Am 2018;45:155-167.

9. Xylinas E, Rink M, Margulis V, Clozel T, Lee RK, Comploj E, Novara G,

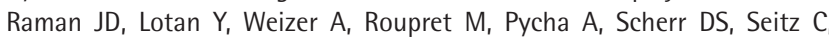
Ficarra V, Trinh OD, Karakiewicz PI, Montorsi F, Zerbib M, Shariat SF; UTUC Collaboration. Impact of renal function on eligibility for chemotherapy and survival in patients who have undergone radical nephro-ureterectomy. BJU Int 2013;112:453-461.

10. Margulis V, Puligandla M, Trabulsi EJ, Plimack ER, Kessler ER, Matin SF, Godoy G, Alva A, Hahn NM, Carducci MA, Hoffman-Censits J; Collaborators. Phase II Trial of Neoadjuvant Systemic Chemotherapy Followed by Extirpative Surgery in Patients with High Grade Upper Tract Urothelial Carcinoma. J Urol 2020;203:690-698.

11. Leow JJ, Chong KT, Chang SL, Bellmunt J. Upper tract urothelial carcinoma: a different disease entity in terms of management. ESMO Open 2017;1:e00126.
12. Audenet $F$, Yates DR, Cussenot 0 , Rouprêt $M$. The role of chemotherapy in the treatment of urothelial cell carcinoma of the upper urinary tract (UUTUCC). Urol Oncol 2013;31:407-413.

13. Kim DK, Lee JY, Kim JW, Hah YS, Cho KS. Effect of neoadjuvant chemotherapy on locally advanced upper tract urothelial carcinoma: A systematic review and meta-analysis. Crit Rev Oncol Hematol 2019;135:59-65.

14. Foerster B, Abufaraj M, Petros F, et al. Efficacy of Preoperative Chemotherapy in High Risk Upper Tract Urothelial Carcinoma. J Urol 2000;203:1101-1108.

15. Oosterlinck W, Solsona E, van der Meijden AP, Sylvester R, Böhle A, Rintala E, Lobel $B$; European Association of Urology. EAU guidelines on diagnosis and treatment of upper urinary tract transitional cell carcinoma. Eur Urol 2004:46:147-154.

16. Tse J, Ghandour R, Singla N, Lotan Y. Molecular predictors of complete response following neoadjuvant chemotherapy in urothelial carcinoma of the bladder and upper tracts. Int J Mol Sci 2019;20:793.

17. Meng X, Chao B, Vijay V, Silver H, Margolin EJ, Balar A, Taneja SS, Shah O, Bjurlin MA, Anderson CB, Huang WC. High Response Rates to Neoadjuvant Chemotherapy in High-Grade Upper Tract Urothelial Carcinoma. Urology 2019:146-152.

18. Fahmy NM, Mahmud S, Aprikian AG. Delay in the surgical treatment of bladder cancer and survival: systematic review of the literature. Eur Urol 2006;50:1176-1182.

19. Hosogoe $S$, Hatakeyama $S$, Kusaka $A$, Hamano I, Iwamura $H$, Fujita $N$ Yamamoto $H$, Tobisawa $Y$, Yoneyama $T$, Yoneyama $T$, Hashimoto $Y$, Koie T, Ohyama C. Platinum-based Neoadjuvant Chemotherapy Improves Oncological Outcomes in Patients with Locally Advanced Upper Tract Urothelial Carcinoma. Eur Urol Focus 2018;4:946-953.

20. Kubota $Y$, Hatakeyama S, Tanaka T, Fujita N, Iwamura H, Mikami J, Yamamoto $H_{\text {, Tobisawa }}$, Yoneyama T, Yoneyama T, Hashimoto $Y$, Koie T, Ito H, Yoshikawa K, Sasaki A, Kawaguchi T, Ohyama C. Oncological outcomes of neoadjuvant chemotherapy in patients with locally advanced upper tract urothelial carcinoma: a multicenter study. Oncotarget 2017;8:101500101508.

21. NCT02876861 (2016) Neoadjuvant Chemotherapy Versus Surgery Alone in Patients With High-Grade UTUC. Https://clinicaltrials.gov/show/ nct02876861

22. Neoadjuvant Upper Tract Invasive Cancer Trial (NAUTICAL) Full Text View - ClinicalTrials.gov. https://clinicaltrials.gov/ct2/ show/NCT04574960?term =neoadjuvant+chemotherapy\&tcond $=$ Urothelial+Carcinomactdraw=2Ctrank=7. Accessed 2 Nov 2020.

23. Vale CL. Neoadjuvant chemotherapy in invasive bladder cancer: update of a systematic review and meta-analysis of individual patient data advanced bladder cancer (ABC) meta-analysis collaboration. Eur Urol 2005;48:202205. 